\title{
A search for clusters and groups of galaxies on the line of sight towards 8 lensed quasars ${ }^{\star}$
}

\author{
C. Faure ${ }^{1}$, D. Alloin ${ }^{2,4}$, J. P. Kneib ${ }^{3,5}$, and F. Courbin ${ }^{6}$ \\ 1 Universidad Católica de Chile, Departamento de Astronomia y Astrofisica, Casilla 306, Santiago 22, Chile \\ e-mail: cfaure@astro.puc.cl \\ 2 European Southern Observatory, Alonso de Cordova 3107, Casilla 19001, Santiago 19, Chile \\ 3 Observatoire Midi-Pyrénées, UMR 5572, 14 avenue Édouard Belin, 31400 Toulouse, France \\ ${ }^{4}$ URA2052CNRS/SAp/CEA, L'Orme des Merisiers, 91191 Gif-sur-Yvette, France \\ 5 Caltech Astronomy Department, Mail Code 105-24, Pasadena, CA 91125, USA \\ ${ }^{6}$ Institut d'Astrophysique et de Géophysique, Faculté des Sciences, Université de Liège, Allée du 6 août, 17, B5C, Liège 1, \\ Belgium
}

Received 8 March 2004 / Accepted 19 May 2004

\begin{abstract}
In this paper we present new ESO/VLT FORS1 and ISAAC images of the fields around eight gravitationally lensed quasars: CTQ 414, HE 0230-2130, LBQS 1009-0252, B 1030+074, HE 1104-1805, B 1359+154, H 1413+117 and HE 2149-2745. When available and deep enough, HST/WFPC2 data were also used to infer the photometric redshifts of the galaxies around the quasars. The search of galaxy overdensities in space and redshift, as well as a weak-shear analysis and a mass reconstruction are presented in this paper. We find that there are most probably galaxy groups towards CTQ 414, HE 0230-2130, B 1359+154, H 1413+117 and HE 2149-2745, with masses $\leq 4 \times 10^{14} M_{\odot} \mathrm{h}^{-1}$. Considering its photometric redshift, the galaxy group discovered in the field around HE 1104-1805 is associated with the quasar rather than with the lensing potential.
\end{abstract}

Key words. galaxies: clusters: general - gravitational lensing

\section{Introduction}

Gravitational lensing of distant quasars is a powerful tool for addressing important cosmological and astrophysical questions. These include the distribution of dark matter in galaxies (Keeton et al. 1998), the determination of the Hubble parameter $H_{0}$ through the measurement of the time delay between the quasar image light curves (Refsdal 1964), as well as ultimately the measurement of the value of the cosmological constant $\Lambda$ (Kochanek 1996). All the complexity in resolving these questions is due to the difficulty in modeling the lensing potential. Until now, most of the multiply imaged quasars were thought to be lensed by one main and single lensing galaxy, disregarding the local environment. Yet, while modeling these systems, it has been realized that the addition of an "external shear" contribution is mandatory in many cases to reproduce the observations (Keeton et al. 1997). It is now known that in at least one quarter of the cases the lensing potential is much more complex than a single lensing galaxy and that it is associated with

* Based on observations obtained with VLT/ANTU at ESO-Paranal Observatory (programs 67.A-0502 and 69.A-0247) and with the Hubble Space Telescope, operated by NASA. a galaxy cluster or a galaxy group. Actually, the first X-ray detection of galaxy groups associated with the lensing potential in quadruple quasars has been made recently towards the quasars RX J0911+0551 (Morgan et al. 2001), Q 0957+561 (Chartas et al. 2002), PG $1115+80$ and B 1422+231 (Grant et al. 2003). Optically, many galaxy clusters or groups have been discovered in the line of sight towards gravitationally lensed quasars like PG 1115+80 (Kundic et al. 1997), Q 0957+561 (AngoninWillaine et al. 1994), the Cloverleaf (Kneib et al. 1998b), RX J0911+0551 (Kneib et al. 2000) and SBS 1520+530 (Faure et al. 2002).

In this paper we present the new VLT/FORS1 and ISAAC observations and wide-field analysis of eight gravitationally lensed quasars in the southern sky: CTQ 414, HE 0230-2130, LBQS 1009-0252, B 1030+074, HE 11041805, B 1359+154, H 1413+117 and HE 2149-2745. In previous studies of these systems, the modeling of the lensing potential suffered from a lack of observational constraints and a poor knowledge of the quasar image surroundings. The modeling led indeed to the conclusion that the contribution of an external shear was mandatory for most of these cases for reproducing the quasar image configuration using a realistic model for 
Table 1. Properties of the lensed quasar sample. Column 1: quasar name. Columns 2, 3: right ascension and declination of quasar image A (J2000). Column 4: quasar redshift. Column 5: lensing galaxy redshift, where "?" means "unknown" and "(p)" means "photometric redshift". Column 6: size of the system corresponding to the maximal angular separation between the quasar images (in arcsecond). Column 7: system configuration. Column 8: predicted value of the external shear strength and position angle of the shear ${ }^{1}$ Lehár et al. (2002), ${ }^{2}$ Saha \& Williams (2003), ${ }^{3}$ Lopez et al. (1998), ${ }^{4}$ Rusin et al. (2001). The symbol "-" means that the system has never been modeled.

\begin{tabular}{cllllllll}
\hline \hline Quasar & RA & Dec & $z_{\text {qso }}$ & $z_{\text {lens }}$ & Size & Configuration & Ext. shear and & PA(deg) \\
\hline CTQ 414 & $01^{\mathrm{h}} 58^{\mathrm{m}} 41.43^{\mathrm{s}}$ & $-43^{\circ} 25^{\prime} 3.4^{\prime \prime}$ & 1.29 & $?$ & $1.22^{\prime \prime}$ & double & - & \\
HE 0230-2130 & $02^{\mathrm{h}} 32^{\mathrm{m}} 33.1^{\mathrm{s}}$ & $-21^{\circ} 17^{\prime} 26^{\prime \prime}$ & 2.16 & $\leq 1.6$ & $2.10^{\prime \prime}$ & quadruple & - & \\
LBQS 1009-0252 & $10^{\mathrm{h}} 12^{\mathrm{m}} 15.71^{\mathrm{s}}$ & $-03^{\circ} 07^{\prime} 02^{\prime \prime}$ & 2.74 & $0.88(\mathrm{p})$ & $1.54^{\prime \prime}$ & double & $0.053^{1}$ & -36 \\
B 1030+074 & $10^{\mathrm{h}} 33^{\mathrm{m}} 34.08^{\mathrm{s}}$ & $+07^{\circ} 11^{\prime} 25.5^{\prime \prime}$ & 1.54 & 0.599 & $1.56^{\prime \prime}$ & double & $0.09^{1}$ & -39 \\
HE 1104-1805 & $11^{\mathrm{h}} 06^{\mathrm{m}} 33.45^{\mathrm{s}}$ & $-18^{\circ} 21^{\prime} 24.2^{\prime \prime}$ & 2.32 & 0.73 & $3.19^{\prime \prime}$ & double & $0.05^{1}$ & -38 \\
B 1359+154 & $14^{\mathrm{h}} 01^{\mathrm{m}} 35.55^{\mathrm{s}}$ & $+15^{\circ} 13^{\prime} 25.6^{\prime \prime}$ & 3.235 & $?$ & $1.71^{\prime \prime}$ & sextuple & 0.12 to $0.26^{4}$ & 71 to 97 \\
H 1413+117 & $14^{\mathrm{h}} 15^{\mathrm{m}} 46.40^{\mathrm{s}}$ & $+11^{\circ} 29^{\prime} 41.4^{\prime \prime}$ & 2.55 & $0.9(\mathrm{p})$ & $1.10^{\prime \prime}$ & quadruple & $0.08^{2}$ & 70 \\
HE 2149-2745 & $21^{\mathrm{h}} 52^{\mathrm{m}} 07.44^{\mathrm{s}}$ & $-27^{\circ} 31^{\prime} 50.2^{\prime \prime}$ & 2.03 & $0.5(\mathrm{p})$ & $1.71^{\prime \prime}$ & double & $>0.21^{3}$ & 58 \\
\hline
\end{tabular}

the main lensing galaxy. In this paper, we first provide a brief summary of former studies for each system (Sect. 2). Then we describe the new ground-based VLT/UT1 observations, and the HST dataset retrieved from the archive (Sect. 3). In Sect. 4 we present and discuss the results of our search for galaxy overdensities, and in Sect. 5 we provide the galaxy photometric redshifts. In Sect. 6 we present the weak-shear analyses and the mass reconstructions performed from the deep FORS1 images. And finally, in Sect. 7, we provide final remarks and present our conclusions. We adopt $H_{0}=65 \mathrm{~km} \mathrm{~s}^{-1} \mathrm{Mpc}^{-1}, \Omega=0.3$ and $\Lambda=0.7$ throughout the paper.

\section{Lens sample}

The goal of this project is to map the line of sight towards a large number of lensed quasars in the southern sky, up to the redshift of the lens. Indeed, the systems studied in this paper have not yet been modeled in a satisfactory manner due to the lack of constraints on the line of sight distribution towards the quasar. In particular, little information is available for characterizing the external shear due to possible intervening galaxy clusters or groups at high redshift along their line of sight.

In this section, we present a brief summary of the previous studies performed on these systems. The quasar coordinates and redshifts, as well as some characteristics of the lensing potential are given in Table 1 (from CfA-Arizona Space Telescope Lens Survey ${ }^{1}$, CASTLES; Muñoz et al. 1998).

As in most papers about gravitationally lensed quasars, we will use the convention that the brightest quasar image (at the date and in the filter of the discovery) is labeled A, the second brightest one is labeled $\mathrm{B}$, etc.

\subsection{CTQ 414}

CTQ 414 (also named Q J0158-4325) has been first observed in the Calán-Tololo Survey (CTS; Maza et al. 1995), and was identified as a doubly imaged quasar at redshift $z=1.29$ by Morgan et al. (1999). The quasar images are separated

\footnotetext{
1 Gravitational Lens Database: http://cfa-www.harvard.edu/castles/
}

by $1.22^{\prime \prime}$. A PSF subtraction of the quasar images reveals an extended object lying between the quasar images: it is thought to be the lensing galaxy. It is located $0.72^{\prime \prime}$ West of image A. The presence of the lensing galaxy has been confirmed by HST NICMOS observations (CASTLES). No mass model has been proposed for this system until now.

\subsection{HE 0230-2130}

HE 0230-2130 was discovered in the course of the Hamburg/ESO survey (Wisotzki et al. 1996), and confirmed to be a lensed quasar by Wisotzki et al. (1999). It is a quadruply imaged quasar at $z=2.162$, with image angular separations of from $0.7^{\prime \prime}$ to $2.1^{\prime \prime}$. The lensing galaxy was also observed and its apparent magnitude suggests that it is at redshift $z_{l} \leq 1.6$. No mass model has been proposed so far.

\subsection{LBQS 1009-0252}

LBQS 1009-0252 was studied by Surdej et al. (1993) and Hewett et al. (1994). It is a doubly imaged quasar at redshift $z=2.74$. The images are separated by $1.53^{\prime \prime}$ and show strong $\mathrm{Mg}$ II absorption lines at $z=0.869$. A second quasar, quasar C, at redshift $z=1.62$, lies by chance at only $4.6^{\prime \prime}$ from quasar image A. The lensing galaxy was detected, close to quasar image $\mathrm{B}$, in the HST $I$ - and $V$-band frames (Lehàr et al. 2000).

In the lens model of Lehàr et al. (2000), the host galaxy of quasar $\mathrm{C}$ is supposed to produce a shear of $\gamma \sim 0.053$ (after correcting for redshift differences) and, according to these authors, could dominate the external shear the main lensing galaxy is fitted by a Singular Isothermal Ellipsoid (SIE). A more recent model of the lens, requires an external shear: $\gamma=0.017 \pm 0.009$, with an orientation: $\mathrm{PA}_{\gamma}=11 \pm 16 \mathrm{deg}$, if the lensing galaxy is modeled by a Singular Isothermal Sphere (SIS) (Claeskens et al. 2001).

\subsection{B $1030+074$}

The bright radio source B $1030+074$ is a two-component gravitationally lensed system at $z=1.535$ (Xanthopoulos et al. 1998). It was discovered during the Jodrell-Bank VLA 
Astrometric Survey (JVAS, Patnaik et al. 1992; Patnaik 1993; Browne et al. 1998; Wilkinson et al. 1998). The two quasar images are separated by $1.56^{\prime \prime}$. The lensing galaxy was discovered by Fassnacht $\&$ Cohen (1998), at a redshift $z_{l}=0.599$. It shows substructures that could be intrinsic to the galaxy or that could trace an interacting galaxy system (Jackson et al. 2000).

A first model of the lensing potential was proposed by Xanthopoulos et al. (1998). They modeled the lensing galaxy by an SIE and a flux ratio between the quasar components of 20:1. They estimated the mass of the lensing galaxy within the Einstein ring to be $1.3 \times 10^{11} M_{\odot}$. Then Lehàr et al. (2000) proposed a two-component lens model, the main lensing galaxy $\mathrm{G}$ being modeled by an SIE, and the second one, $\mathrm{G}^{\prime}$, modeled by an SIS. They concluded that, without external shear, the orientation of the SIE is inconsistent with that observed for the light of galaxy $\mathrm{G}$, whereas it provides a good fit of the lens constraints. This disagreement is still unresolved.

\subsection{HE 1104-1805}

HE 1104-1805 (Wisotzki et al. 1993) is a doubly imaged quasar at redshift $z=2.319$, with an image angular separation of 3.19". In 1998 Courbin et al. discovered the lensing galaxy from ground-based near-IR $(J$ - and $K$-band observations). Remy et al. (1998) also detected the lensing galaxy in their WFPC2/I-band image, but not in their $V$-band image. Therefore, they suggested that the lensing galaxy is an early type galaxy. The redshift of the lensing galaxy is $z_{l}=$ 0.729 (Lidman et al. 2000). The recent time-delay measurement (Ofek \& Maoz 2003) gives a value of $\Delta t=-161 \pm 7$ days (68\% confidence level), in disagreement with former results by Gil-Merino et al. $2002(\Delta t=-310 \pm 20$ days $)$ and Pelt et al. 2002 ( $\Delta t=-255$ days). Moreover, this system is known to show conspicuous microlensing effects (Wisotzki et al. 1993; Courbin et al. 2000; Gil-Merino et al. 2002; Schechter et al. 2003), making it more difficult to model. Several mass models have been done (Wisotzki et al. 1998; Courbin et al. 2000; Schechter et al. 2003), all leading to the conclusion that the contribution of an external shear is mandatory for reproducing simultaneously the quasar image configuration, the time-delay and the flux ratios, using a realistic ellipticity and mass for the lensing galaxy.

\subsection{B $1359+154$}

The complex lensed system B 1359+154 consists of a set of six images of a quasar at $z=3.235$ (Rusin et al. 2001). The main lensing potential is due to a group of three galaxies at $z \sim 1$. Rusin et al. have assumed that the true number of lensed quasar images is nine, and that only six of them are amplified and detected. They derived mass models, assuming four different galaxy potentials for the three main lensing galaxies: SIS, SIE, Pseudo-Jaffe spherical (PJS) and Pseudo-Jaffe ellipticals (PJE) (for the definitions, see Keeton \& Kochanek 1998). None of these models matches well the flux ratios and the relative distances between the quasar images. To be correctly modeled, the complexity of this system requires more observational constraints.

\section{7. $H 1413+117$}

The Cloverleaf is a distant quasar at $z=2.558$ discovered by Hazard et al. (1984) and identified as a lensed quasar by Magain et al. (1988). H 1413+117 shows four images, with angular separations from $0.76^{\prime \prime}$ to $1.1^{\prime \prime}$. The lensing galaxy was discovered by Kneib et al. (1998b) in the NICMOS-2/F160w observations. A galaxy overdensity was also discovered in the WFPC2/I- and $R$-band (filter $I$ : F814w, filter $R$ : F702w) images, in the magnitude ranges $23 \leq I<25$ and $23 \leq R<25$ (Kneib et al. 1998a,b). According to Kneib et al. (1998a), the colors of the galaxies building the overdensity suggest a redshift of $z=0.9$. Several models have been proposed for the lensing object, using also constraints from the CO-map of the quasar images (Kneib et al. 1998b), and the ones which reproduce the observations best are those that take into account the galaxy overdensity discovered by Kneib et al. (1998a,b).

\subsection{HE 2149-2745}

The doubly imaged quasar HE 2149-2745 at redshift $z=2.033$ presents an angular separation of 1.71" (Wisotzki et al. 1998). The lensing galaxy, appearing after PSF subtraction of the quasar images, has an $R$-band magnitude $R=20.4$. If the lensing galaxy is elliptical, its $V-R$ corresponds to a maximum redshift of 0.5 . The lensing galaxy lies at almost equal projected distance from both quasar images, and the line connecting the images coincides with the major axis of the lensing galaxy. Lopez et al. (1998) proposed a model for the lens. They used a singular isothermal elliptical mass distribution (SIEMD, Kassiola \& Kovner 1993). Due to the singular geometrical configuration of the quasar images, an external shear contribution is mandatory in the model. Because of the non-detection of any galaxy cluster in the field (magnitude limit: $R \sim 23$ ), Lopez et al. imposed the external shear $\gamma$ to be less than 0.21 . Their best models give a lens mass $M=1.5(2.4) \times 10^{11} M_{\odot}$ for a lensing galaxy at redshift $z_{l}=0.3(0.5)$, corresponding to a mass to light ratio of $\mathcal{M} / \mathcal{L}_{R}=5(2)$. A time delay between the light curves of the quasar images has been measured of $\Delta t=103 \pm 12$ days (Burud et al. 2002).

\section{Observations - data reduction}

\subsection{Ground-based dataset}

We took deep and high resolution FORS1 $R$-band images to be able to exploit wide field images in the analysis of the lensing potential on large scale, performing a galaxy overdensity search and a weak-shear analysis. The near-IR images taken with the ISAAC instrument were mandatory to trace the stellar mass of galaxies at high redshift $(z \sim 3-4)$, and to measure galaxy photometric redshifts from multi-color analysis.

The ground-based observations consist of two datasets taken with ISAAC (Cuby et al. 2002) and with FORS1 (Szeifert 2002) attached to the $8.2 \mathrm{~m}$ telescope VLT/ANTU, 
Table 2. FORS1 and ISAAC observations. Column 1: quasar name. Column 2: filter. Column 3: total exposure time in ks. Column 4: field of view used for the analysis.

\begin{tabular}{cllll}
\hline \hline Quasar & Band & Seeing & Exp. time & Field \\
\hline CTQ 414 & $R$ & $0.50^{\prime \prime}$ & 1.45 & $6.5^{\prime} \times 6.6^{\prime}$ \\
& $J$ & $0.51^{\prime \prime}$ & 2.00 & $2.3^{\prime} \times 2.4^{\prime}$ \\
& $K \mathrm{~s}$ & $0.50^{\prime \prime}$ & 1.50 & $2.2^{\prime} \times 2.2^{\prime}$ \\
\hline HE 0230-2130 & $R$ & $0.74^{\prime \prime}$ & 3.40 & $6.4^{\prime} \times 6.6^{\prime}$ \\
& $J$ & $0.58^{\prime \prime}$ & 2.60 & $2.5^{\prime} \times 2.4^{\prime}$ \\
& $K \mathrm{~s}$ & $0.50^{\prime \prime}$ & 2.70 & $2.1^{\prime} \times 2.4^{\prime}$ \\
\hline LBQS 1009-0252 & $R$ & $0.72^{\prime \prime}$ & 3.90 & $6.5^{\prime} \times 6.6^{\prime}$ \\
& $J$ & $0.44^{\prime \prime}$ & 3.80 & $2.4^{\prime} \times 2.5^{\prime}$ \\
& $K$ & $0.53^{\prime \prime}$ & 4.50 & $2.2^{\prime} \times 2.2^{\prime}$ \\
\hline B 1030+074 & $R$ & $0.48^{\prime \prime}$ & 6.00 & $6.6^{\prime} \times 6.6^{\prime}$ \\
& $J$ & $0.60^{\prime \prime}$ & 3.60 & $2.4^{\prime} \times 2.4^{\prime}$ \\
& $K \mathrm{~s}$ & $0.60^{\prime \prime}$ & 4.80 & $2.3^{\prime} \times 2.3^{\prime}$ \\
\hline HE 1104-1805 & $R$ & $0.66^{\prime \prime}$ & 0.80 & $6.6^{\prime} \times 6.7^{\prime}$ \\
& $J$ & $0.51^{\prime \prime}$ & 3.50 & $2.4^{\prime} \times 2.4^{\prime}$ \\
& $K \mathrm{~s}$ & $0.34^{\prime \prime}$ & 4.80 & $2.2^{\prime} \times 2.3^{\prime}$ \\
\hline B 1359+154 & $R$ & $0.62^{\prime \prime}$ & 4.80 & $6.6^{\prime} \times 6.7^{\prime}$ \\
& $J$ & $0.64^{\prime \prime}$ & 3.60 & $2.3^{\prime} \times 2.3^{\prime}$ \\
& $K \mathrm{~s}$ & $0.47^{\prime \prime}$ & 4.50 & $2.4^{\prime} \times 2.4^{\prime}$ \\
\hline H 1413+117 & $R$ & $0.58^{\prime \prime}$ & 1.70 & $6.2^{\prime} \times 6.2^{\prime}$ \\
& $J$ & $0.59^{\prime \prime}$ & 2.60 & $2.6^{\prime} \times 2.6^{\prime}$ \\
& $K \mathrm{~s}$ & $0.49^{\prime \prime}$ & 3.60 & $2.6^{\prime} \times 2.6^{\prime}$ \\
\hline HE 2149-2745 & $R$ & $0.57^{\prime \prime}$ & 1.70 & $6.5^{\prime} \times 6.7^{\prime}$ \\
& $J$ & $0.48^{\prime \prime}$ & 2.60 & $2.3^{\prime} \times 2.5^{\prime}$ \\
& $K$ & $0.47^{\prime \prime}$ & 3.60 & $2.3^{\prime} \times 2.5^{\prime}$ \\
\hline & & & &
\end{tabular}

at ESO/Paranal. The imaging datasets were obtained during ESO periods 67 and 69 (programs 67.A-0502(A, B, C) and 69.A-0247(A, B, C)). The ISAAC images $(1024 \times 1024$ pixels, $0.1484^{\prime \prime}$ per pixel) were taken in two near-IR filters: the $J$ and $K$ s-filters. We reduced the data following standard procedures (ISAAC Data Reduction Guide, Amico et al. 2002). All the images ( $J$ - and $K$ s-bands) taken in P67 were affected by the "odd-even column effect", arising from an ISAAC misfunctioning during this period (this concerns objects CTQ 414, HE 0230-2130, H 1413+117 and HE 2149-2745). This artifact was successfully removed through a Fourier analysis procedure (see ISAAC Handbook, Cuby et al. 2002). When images of a same object were taken during different nights we have reduced all images to the same "seeing", through convolution by a Gaussian, before combining them. A summary of the observations is given in Table 2.

The FORS1 $R$-band images $\left(2040 \times 2040\right.$ pixels, $0.2^{\prime \prime}$ per pixel) were reduced using sky flat-fields and standard IRAF procedures for optical image reduction. Saturated stars were masked to perform the sky subtraction and the galaxy extraction (both done using SExtractor; Bertin \& Arnouts 1996). Quantitative information on the quality of the images is provided in Table 2.

The sizes and positions of the ISAAC fields relative to the FORS1 fields are shown in Figs. 3 and 4.

As shown in Table 2, the total exposure time varied from one system to the other, a consequence of the observations being conducted in service mode. In particular, some systems are
Table 3. WFPC2 observations. Column 1: quasar name. Column 2: filter. Column 3: proposal P.I. and proposal ID. Column 4: total exposure time (in ks).

\begin{tabular}{clll}
\hline \hline Quasar & Band & P.I and ID & Exp. Time \\
\hline CTQ 414 & $F 555 w$ & Falco (9133) & 1.60 \\
& $F 814 w$ & Impey (8268) & 2.00 \\
\hline HE 1104-1805 & $F 814 w$ & Surdej (5958) & 1.00 \\
\hline B 1359+154 & $F 555 w$ & Impey (8268) & 5.20 \\
& $F 814 w$ & Impey (8268) & 5.00 \\
\hline H 1413+117 & $F 814 w$ & Turnshek (5621) & 5.20 \\
\hline HE 2149-2745 & $F 555 w$ & Falco (8175) & 1.60 \\
& $F 814 w$ & Falco (7495) & 1.60 \\
\hline
\end{tabular}

under-exposed, like HE 1104-1805 in the R-band and CTQ 414 in the $K$ s-band.

\subsection{HST imaging}

We have searched the HST public archive for additional images of the quasar fields around: CTQ 414, HE 0230-2130, HE 1104-1805, B 1359+154, H 1413+117 and HE 2149-2745, in the $I$ - and $V$-bands ( $F 814 w$ and $F 555 w$ ). Details of the observations are given in Table 3 . In most cases we are able to use directly the WFPC2 association frames ${ }^{2}$, otherwise we used standard reduction procedures from the IRAF/STSDAS package. To project the HST images onto the sky we used the SWarp package from Bertin ${ }^{3}$.

\section{Looking for galaxy overdensities}

\subsection{Photometry and galaxy-star separation}

We have searched for galaxy number overdensities in the field around the quasar images. If these overdensities trace galaxy clusters or galaxy groups, they must be included in the total lensing potential.

The extraction and photometry of the objects from all frames were performed using the SExtractor software (Bertin \& Arnouts 1996). Galaxies are separated from stars using a combination of FWHM (Full Width-Half Maximum) versus magnitude (MAG_BEST in SExtractor) analysis and $\mu_{\max }$ (peak surface brightness in mag $\operatorname{arcsec}^{-2}$ ) versus magnitude analysis (see Bardeau et al. 2004). The stars are the brightest objects of the catalogs, having the highest peak surface brightness and the smallest FWHM.

\subsection{Overdensities above the galaxy background level}

To search for galaxy number overdensities, we have proceeded as follows: for each field, the galaxy magnitude catalogs were split in magnitude slices of one magnitude width, allowing a first-order study of different redshift slices. For each magnitude slice, we cut the image into a grid of $10 \times 10$ cells ${ }^{4}$. For

\footnotetext{
${ }^{2}$ http://archive.eso.org/wdb/wdb/hst/asn_wfpc2/form

3 http://terapix.iap.fr/complement/oldSite/ soft/swarp/

$4 \sim 40^{\prime \prime}$ size cell in the $R$-band image, $15^{\prime \prime}$ in the $J$ - and $K$ s-images.
} 
Table 4. Characteristics of the galaxy number overdensities. Column 1: quasar name. Column 2: limit for the galaxy catalog (said to be as complete as the FDF galaxy catalog where the curves in Fig. 1-top join). Column 3: magnitude range over which we can study the significance of the galaxy overdensity. Column 4: position of the center of the galaxy number overdensity relative to quasar image A (see Figs. 3 and 4). The uncertainty of the exact position of the galaxy overdensity is about $5^{\prime \prime}$. Column 5: galaxy density at the location of the galaxy overdensity center. Column 6: FDF mean galaxy density in the magnitude range given in Col. 3. Column 7: FDF maximal galaxy density in the same magnitude range. Column 8: detection level (in $\sigma$ ) of the cluster compared to the FDF mean galaxy density level. Column 9: detection level of the cluster (in the magnitude range given in parentheses) above the galaxy background level measured in the same frame.

\begin{tabular}{|c|c|c|c|c|c|c|c|c|}
\hline & $\begin{array}{c}\text { Compl. } \\
\text { limit }\end{array}$ & $\begin{array}{c}\text { Mag } \\
\text { range }\end{array}$ & $\begin{array}{c}\text { Galaxy overdensity } \\
\text { location } \\
\Delta \alpha, \Delta \delta\end{array}$ & $\begin{array}{l}\text { Density } \\
\text { gal } /\left({ }^{\prime}\right)^{2}\end{array}$ & $\begin{array}{r}\text { FDF } \\
\text { mean } \\
\text { density }\end{array}$ & $\begin{array}{r}\text { FDF } \\
\text { max } \\
\text { density }\end{array}$ & $\begin{array}{r}\text { Above } \\
\text { FDF } \\
\text { density }\end{array}$ & $\begin{array}{r}\text { Above } \\
\text { background } \\
\text { mean }\end{array}$ \\
\hline CTQ 414 & 25.2 & $24 \leq R<25$ & $-10^{\prime \prime},-05^{\prime \prime}$ & 29.0 & 14.4 & 25.0 & $2.6 \sigma$ & $4 \sigma(24 \leq R<25)$ \\
\hline HE 0230-2130 & 24.5 & $24 \leq R<24.5$ & $+40^{\prime \prime},-05^{\prime \prime}$ & 7.7 & 5.4 & 14.8 & $1.1 \sigma$ & $2.5 \sigma(24 \leq R<25)$ \\
\hline LBQS 1009-0252 & 24.0 & $23 \leq R<24$ & $+60^{\prime \prime},+40^{\prime \prime}$ & 10.8 & 5.2 & 10.0 & $2.8 \sigma$ & $3.5 \sigma(23 \leq R<25)$ \\
\hline B $1030+074$ & 24.0 & $21 \leq R<23$ & $-90^{\prime \prime},-70^{\prime \prime}$ & 11.0 & 3.2 & 7.0 & $5.6 \sigma$ & $4.5 \sigma(21 \leq R<23)$ \\
\hline HE 1104-1805 & 23.3 & $23 \leq R<23.3$ & $00^{\prime \prime},+50^{\prime \prime}$ & 9.0 & 1.2 & 2.0 & $26 \sigma$ & $4 \sigma(23 \leq R<24)$ \\
\hline B $1359+154$ & 24.3 & $22 \leq R<23$ & $-08^{\prime \prime},+03^{\prime \prime}$ & 4.6 & 2.2 & 6.0 & $3.0 \sigma$ & $5 \sigma(22 \leq R<23)$ \\
\hline H $1413+117$ & 25.0 & $23 \leq R<25$ & $-10^{\prime \prime},-10^{\prime \prime}$ & 38.3 & 19.7 & 32.5 & $2.3 \sigma$ & $5 \sigma(23 \leq R<25)$ \\
\hline HE 2149-2745 & 24.0 & $23 \leq R<24$ & $+08^{\prime \prime},+07^{\prime \prime}$ & 10.6 & 5.2 & 10.0 & $2.6 \sigma$ & $6 \sigma(23 \leq R<25)$ \\
\hline
\end{tabular}

each galaxy $G_{1}$ in a cell we have measured the distance between $G_{1}$ and its ninth closest galaxy $G_{10}$. Then, we have calculated the area, $\mathcal{A}$, covered by a circle of radius $\mathcal{R}=\left|G_{1}-G_{10}\right|$. By definition, the number of galaxies included in the area $\mathcal{A}$, with radius $\mathcal{R}=\left|G_{1}-G_{10}\right|$, is 10 . Therefore, the galaxy number density associated to the galaxy $G_{1}$ is the ratio $10 / \mathcal{A}$. A mean galaxy density for each cell is then derived, averaging the densities associated to all galaxies in the cell. The grid is chosen considering that the central part of a galaxy cluster would cover between 1 and 4 cells, depending on its redshift and on its central density. Then the isodensity contours of the number of galaxies per $\operatorname{arcmin}^{2}$ across the field are drawn: when an overdensity is observed in two consecutive magnitude slices, the two sub-catalogs are added to increase the signal to noise of the overdensity detection. The results are displayed in Figs. 3 and 4.

To evaluate the significance of these detections we have performed the following tests. As mentioned above, a galaxy cluster should appear in one to four contiguous cells that are overdense in comparison to the rest of the frame. We have plotted the number of cells versus the galaxy density in the cells. This plot can be fitted by a Gaussian (see Faure et al. 2003 for a similar analysis), providing the mean galaxy density in a field for a given magnitude range, as well as a standard deviation $\sigma$ (Figs. 3 and 4). This estimator of detection is given in Col. 9 of Table 4 and results are discussed in Sect. 4.4.

\subsection{Comparison with the FORS Deep field}

For the $R$-band images we have also used the FORS Deep Field $^{5}$ (FDF; Heidt et al. 2003) $R$-band galaxy catalog as a reference field. The $R$-band data set of the FDF covers an area of $\sim 7.4^{\prime} \times 7.4^{\prime}(26.4$ kilo-second exposure time $)$, up to $R=26.7$ (50\% completeness). The FDF field is centered close to the South Galactic Pole $\left(1^{\mathrm{h}} 6^{\mathrm{m}} 3.6^{\mathrm{s}},-25^{\circ} 45^{\prime} 46^{\prime \prime}, \mathrm{J} 2000\right)$. It has been

\footnotetext{
5 The FDF photometric catalog is available from: http://cdsweb.u-strasbg.fr/cgi-bin/qcat?J/A+A/398/49
}

chosen because there was no previously known galaxy cluster in this field.

The FDF dataset is much deeper than our dataset (see Fig. 1). So we are allowed to compare them up to the magnitude corresponding to the $100 \%$ completeness limit of our dataset. These magnitudes are displayed in Table 4.

We have proceeded as follows: first, we split the FDF catalog in magnitude ranges identical to those where galaxy overdensities were discovered in the fields around the quasars. When an overdensity was detected in a magnitude range such that the upper magnitude was higher than the completeness magnitude, we have compared only the part of the catalog for which the upper magnitude was equal to the completeness magnitude (see Table 4, Cols. 2 and 3). We have partitioned the FDF in a grid of $10 \times 10$ cells. For each cell, a galaxy density in a given magnitude range is measured and as detailed in Sect. 4.2, we have fitted a Gaussian to the histogram describing the "number of cells" versus the "galaxy density". This provides the mean galaxy density, the standard deviation and the maximal density expected in a cluster-free region, in each magnitude range. The results of the comparison between the FDF and our dataset are displayed in Table 4.

\subsection{Results of the search for galaxy overdensities}

The galaxy overdensities detected towards CTQ 414, B 1030+074, HE 1104-1805 and H 1413+117 are well above the FDF mean level, revealing a 2 dimensional structure in the line of sight towards these quasars. On the contrary, the galaxy overdensities discovered towards HE 0230-2130, LBQS 10090252, B 1359+154 and HE 2149-2745 show values lower than (or very close to) the maximal density measured in the FDF field. This could mean that these densities are compatible with the variance of the counts within the field of view. Hence, they may correspond to areas where the count fluctuations reach a slightly higher level, still compatible with random deviation from the mean density. Another possibility is 



Fig. 1. Logarithm of the number of galaxies as a function of magnitude. Top panel: in the FORS1 (R-band) fields around the lensed quasars and in the FORS Deep Field (FDF) Survey (thick solid line; Heidt et al. 2003). All the plots are rescaled to a 6.6 $\times 6.6^{\prime}$ field of view. Middle panel: in the ISAAC (Ks-band) fields around the lensed quasars. Bottom panel: in the ISAAC ( $J$-band) fields around the lensed quasars. Diamonds: B 1359+154; squares: H 1413+117; long-dash line: HE 2149-2745; solid line: HE 1104-1805; dotted line: LBQS 1009-0252; dashed line: B 1030+074; dash-dot line: CTQ 414; dash-dot-dot-dot line: HE 0230-2130.

that there are undetected galaxy groups in the FDF, leading to values of the galaxy densities as strong as in the lens fields.

Comparing the detected overdensity to the mean galaxy density measured over the rest of the frame, we see that, apart from the galaxy overdensities towards HE 0230-2130 and LBQS 1009-0252 (respectively detected at $2.5 \sigma$ and $3.5 \sigma$ above the background level), the overdensities measured towards the other six lensed quasars are all significant $(4 \sigma$ to $6 \sigma)$. Notice that the high detection level $(26 \sigma)$ of the cluster towards HE 1104-1805 compared to the FDF galaxy number density is most probably overestimated. This results from the fact that the galaxy densities are analyzed over a very narrow magnitude range ( $\Delta m=0.3)$, and hence, a small galaxy sample ( $\sim 100$ galaxies), leading consequently to possibly large statistical effects.

Furthermore, there are two cases for which we have detected more than one cluster in the line of sight towards the quasar images. Towards H $1413+117$ a second galaxy 

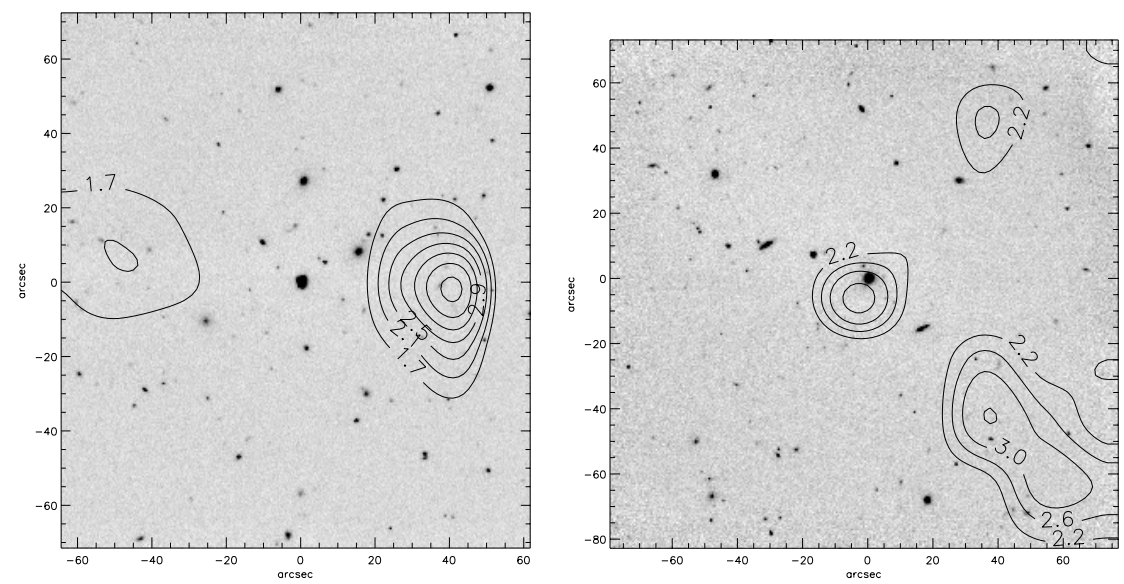

Fig. 2. ISAAC Ks-band images of HE 0230-2130 (left) and $\mathrm{H} 1413+117$ (right). The contours (from $2 \sigma$, increasing by $1 \sigma$ steps) outline regions of equal galaxy number density (label-value attached to each contour in galaxies per $\operatorname{arcmin}^{2}$ ). North is to the top, East to the left. For HE 0230-2130, the galaxy overdensity is located at $\Delta \alpha=40^{\prime \prime}, \Delta \delta=-3^{\prime \prime}$ relative to quasar image A (coordinates $(0,0)$ ) and occurs in the magnitude range $19 \leq K \mathrm{~s}<22$. For $\mathrm{H} 1413+117$, the galaxy overdensity is located at $\Delta \alpha=-3^{\prime \prime}, \Delta \delta=-6^{\prime \prime}$ relative to quasar image $\mathrm{A}$ (coordinates $(0,0))$ in the same magnitude range as for HE 0230-2130.

overdensity has been detected in the magnitude range $25 \leq$ $R<28$, at $3 \sigma$ above the background level (centered $36^{\prime \prime}$ SouthWest to the quasar images). In a similar way, we find a second overdensity towards HE 1104-1805 (78" South-West) in the magnitude range $24 \leq R<25$, at $5 \sigma$ above the background level. But in these two cases, the data used for the estimation extend below the catalog completeness limit, and therefore we cannot assure whether these are real detections or whether they correspond to standard deviations at that level.

In most cases, the number of galaxies detected in the near-IR frames is too low to perform a secure iso-density analysis. Nevertheless, in the Ks-band images towards HE 02302130 and $\mathrm{H} 1413+117$ we find again the galaxy overdensities seen in the $R$-band images (see Fig. 2). The shifts between the positions of the overdensities in the $R$ - and $K$ s-band images come mainly from the use of different cell sizes and locations.

According to these results we conclude that, apart from the marginal galaxy overdensity towards LBQS 1009-0252, all others are possible 3D-structures. That is what we are going to check in the following sections.

\section{Photometric redshifts and color-magnitude analysis}

We can test whether the galaxy overdensities discovered in Sect. 4 are associated to galaxy clusters or galaxy groups by measuring the galaxy photometric redshifts. The redshift will also tell us whether these structures lie in the line of sight towards the quasar or whether they are at the quasar redshift.

\subsection{Photometric redshifts}

Whenever a galaxy overdensity was discovered on the line of sight towards the quasar images, we performed the photometric redshift analysis using the Hyperz software (Bolzonella et al. 2000) and the full dataset available (described in Sect. 3).
The input to Hyperz are the galaxy magnitudes in at least 4 filters ${ }^{6}$. Hyperz performs a comparison between the photometric spectral energy distribution (SED) of the observed galaxy and those derived from a set of reference template spectra, using the same photometric system. Reddening is taken into account using the Calzetti law (Calzetti et al. 2000). We estimate that the current dataset is deep enough to reach giant elliptical galaxies at redshift up to $z=3.5$. Two parameters are important to test the significance of the photometric redshifts found: the $\chi^{2}$ value of the SED fit, and the probability of obtaining this $\chi^{2}: P\left(\chi^{2}\right)$. In the following analysis, we only take into account the redshifts obtained with $\chi^{2} \sim 1$ and $P\left(\chi^{2}\right)>70 \%$, considering that this is the limit of confidence for the redshift determination.

In the FORS1 field around CTQ $\mathbf{4 1 4}$ we have computed the galaxy photometric redshifts using the VLT and HST data. The overlapping field covers only a very small area (see Fig. 3), hence only 7 galaxies have their redshift determined using $5 \mathrm{mag}(V, R, I, J$ and $K \mathrm{~s})$. In addition, we tentatively determined the redshifts of 12 galaxies from their $V-, R-, J$ - and $K$ s-band magnitudes, and of 9 galaxies from their $R-, I-, J$ - and $K$ s-band magnitudes. The results are plotted in Fig. 5, showing a significant peak in redshift at $z=0.5 \pm 0.1$. In the following, this is assumed to be the redshift of the galaxy overdensity discovered towards the quasar.

For HE 0230-2130, we have not been able to determine the photometric redshifts of the galaxies building the observed marginal overdensity, because of the low depth of the WFPC2 images available for this field. However, according to Wisotzki et al. (1999) the lensing galaxy redshift is $z_{l}<1.6$. Assuming that the lensing galaxy is part of the galaxy cluster tentatively discovered, we will use arbitrarily $z=1.0$ for the lensing potential redshift in the mass reconstruction.

For LBQS 1009-0252 there is no significant galaxy overdensity in the field (Sect. 4) and with the galaxy photometry available in only 3 bands $(R, J$ and $K \mathrm{~s})$ we cannot perform a

\footnotetext{
6 A study of the accuracy of the code, according to the number of filters used to infer the redshifts, is detailed in Bolzonella et al. (2000).
} 
H $1413+117$

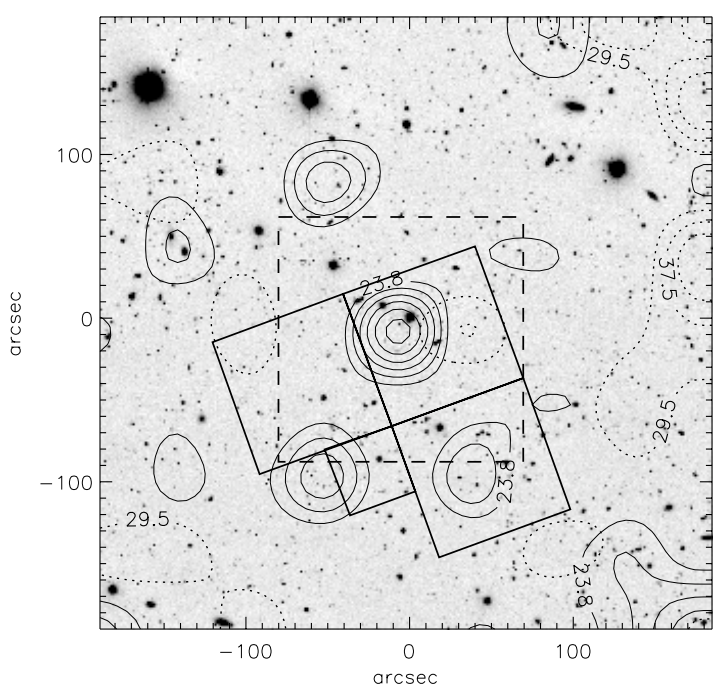

B $1030+074$

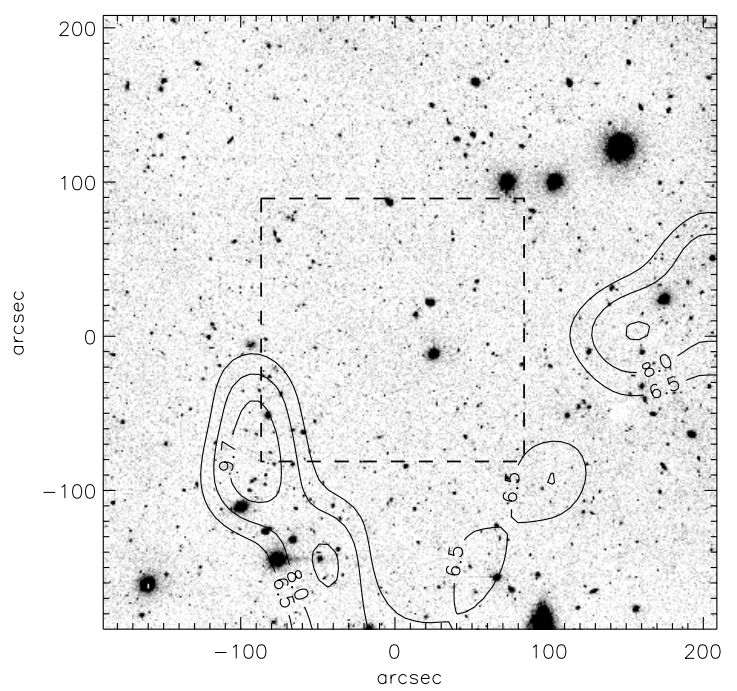

B1359+154

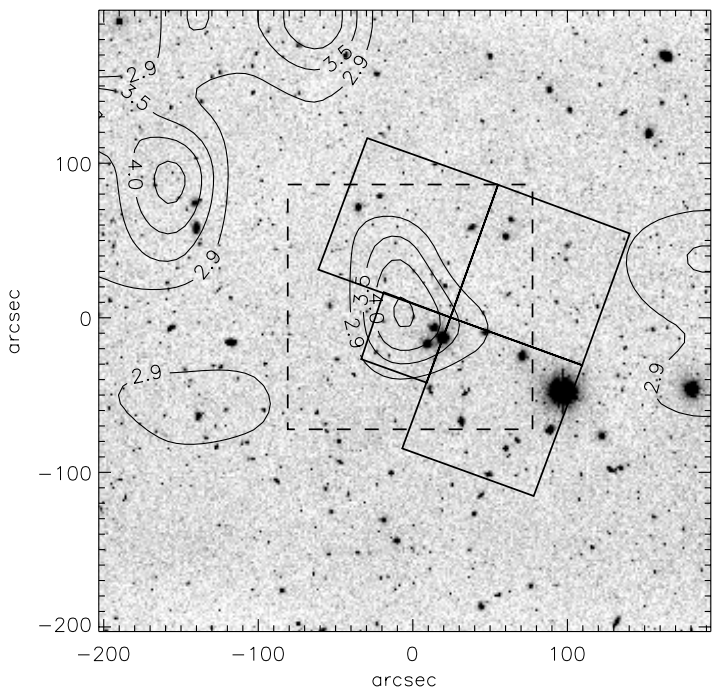

CTQ 414

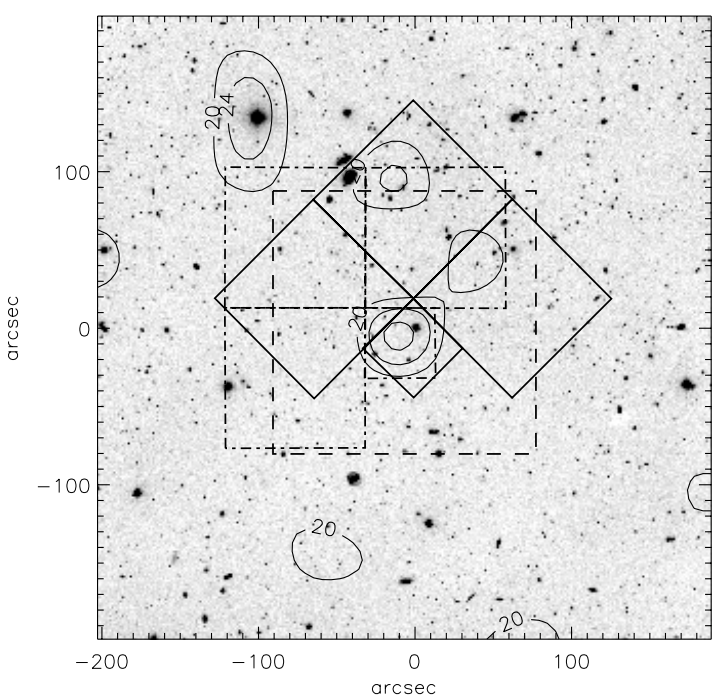

Fig. 3. FORS1 $R$-band images of H 1413+117, B 1359+154, B 1030+074 and CTQ 414. North is to the top, East to the left. The contours (from $2 \sigma$, increasing by $1 \sigma$ ) outline regions of equal galaxy number density (label-value attached to each contour in galaxies per arcmin ${ }^{2}$ ). The dash-line squares represent the position and relative size of the ISAAC field, while the solid-line squares represent the position, size and orientation of the WFPC2 field (in the case of CTQ 414, the dash-point squares represent the position, size and orientation of the WFPC2 $V$-band field, different from the one of the WFPC2 $I$-band ones). For each system, quasar image A is located at coordinates (0, 0). For

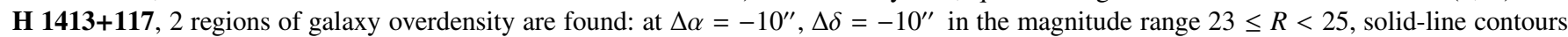
and at $\Delta \alpha=35^{\prime \prime}, \Delta \delta=-5^{\prime \prime}$ in the magnitude range $25 \leq R<28$, dashed-line contours. For $\mathbf{B}$ 1359+154, a galaxy overdensity is found at $\Delta \alpha=-8^{\prime \prime}, \Delta \delta=3^{\prime \prime}(22 \leq R<23)$. For B 1030+074, also one galaxy overdensity is detected $\left(\Delta \alpha=-90^{\prime \prime}, \Delta \delta=-70^{\prime \prime}, 21 \leq R<23\right)$. Finally, for CTQ 414, a galaxy overdensity is discovered in the magnitude range $24 \leq R<25$ at $\Delta \alpha=-10^{\prime \prime}, \Delta \delta=-5^{\prime \prime}$.

photometric redshift analysis. Therefore, a weak-lensing analysis is necessary to define whether or not there is a dark source of shear generating the external-shear value predicted by Lehar et al. (2000, see Table 1).

For B 1030+074, the lack of multi-wavelength data makes impossible photometric redshift measurements. Moreover, the galaxy overdensity detected in the FORS1 field around B 1030+074 (see Sect. 4) is centered outside the ISAAC field. Would this galaxy overdensity be a cluster or a group, it would be too far away from the quasar images $\left(\sim 2^{\prime}\right)$ to play any significant role in the lensing potential (shear strength negligible). Hence, as for LBQS 1009-0252, we expect the weak-shear analysis to conclude whether or not there is a galaxy group or cluster in the line of sight towards the quasar.

Around HE 1104-1805 we have derived the photometric redshifts of the galaxies present in the overlapping HST and VLT fields (see Fig. 4). One WFPC2 chip covers mainly the galaxy overdensity located 50" North to the quasar images. The four galaxies detected both on this chip and on the three VLT images have a redshift in the range: $1.95 \leq z \leq 2.10$. 


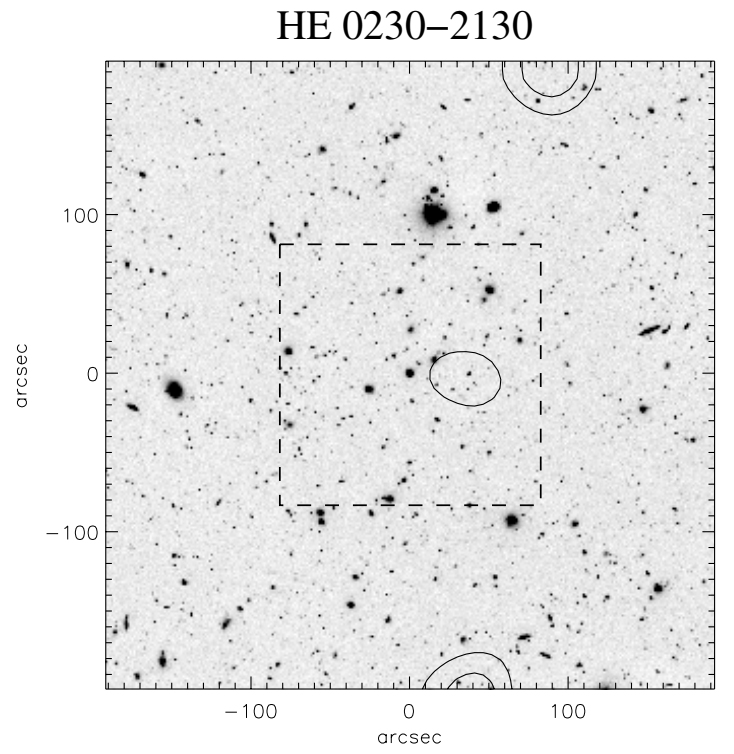

LBQS 1009-0252

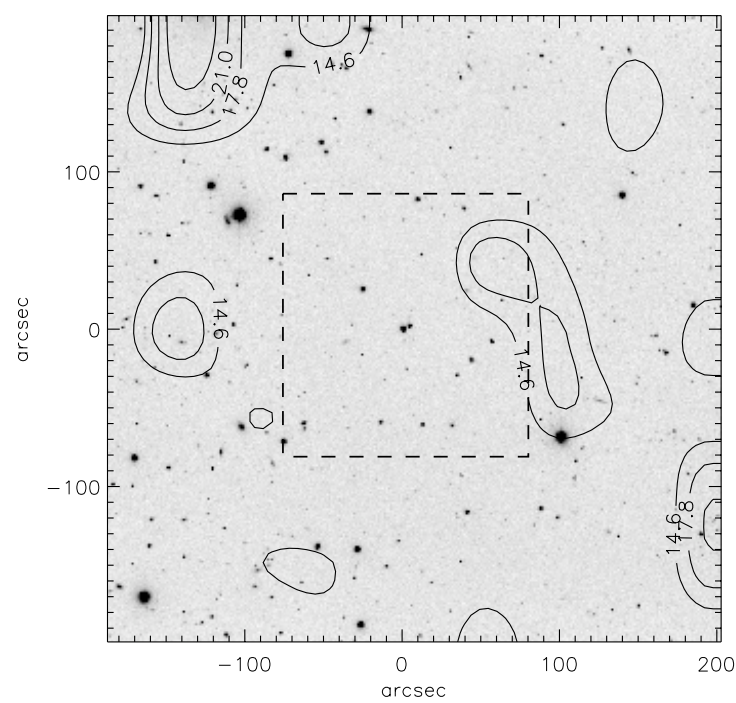

HE 1104-1805

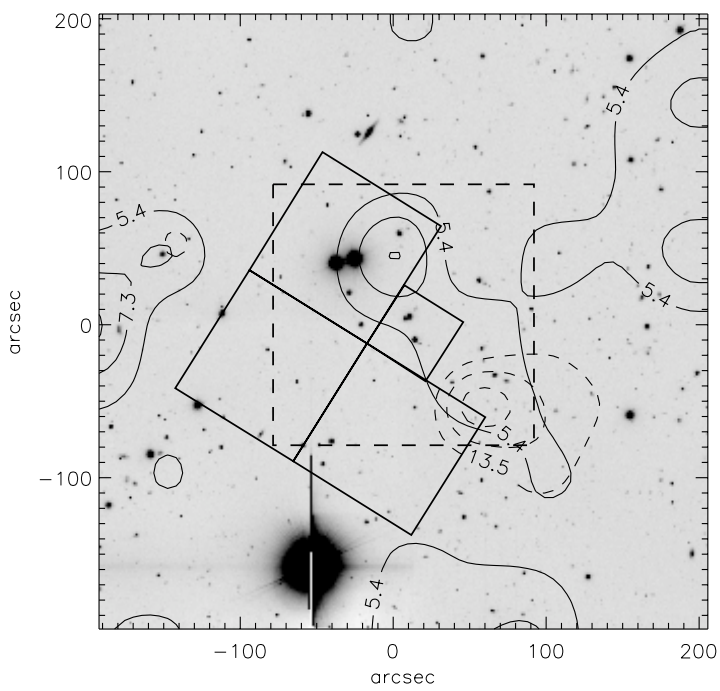

HE 2149-2745

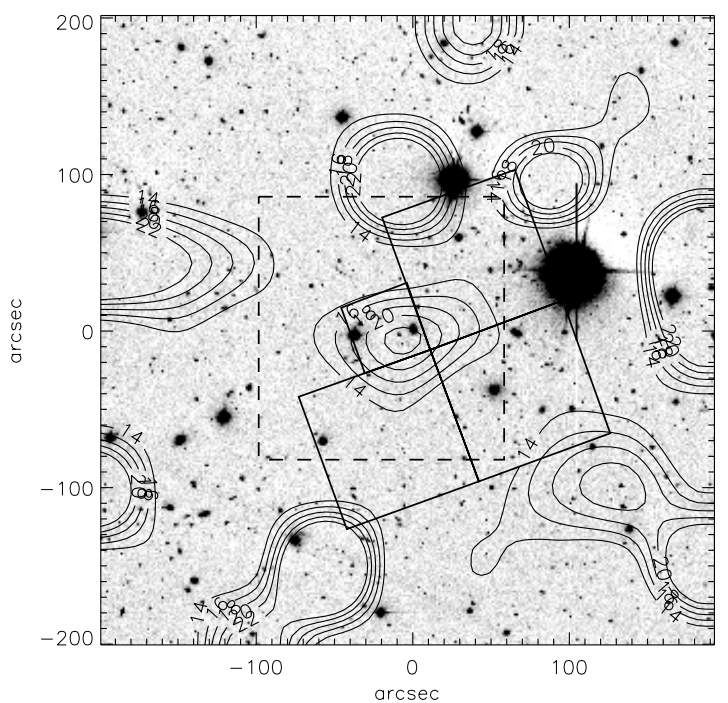

Fig. 4. FORS1 $R$-band images of HE 0230-2130, HE 1104-1805, LBQS 1009-0252 and HE 2149-2745. The contours (from $2 \sigma$, increasing by $1 \sigma$ ) outline regions of equal galaxy number density (label-value attached to each contour in galaxies per $\operatorname{arcmin}^{2}$ ). The dashed-line squares represent the position and relative size of the ISAAC field, while the solid-line squares represent the position, size and orientation of the WFPC2 field. North is to the top, East to the left. For HE 0230-2130, one galaxy overdensity is discovered at $\Delta \alpha=40^{\prime \prime}, \Delta \delta=-5^{\prime \prime}(24 \leq R<25)$. For HE 1104-1805, two galaxy overdensities are discovered: at $\Delta \alpha=0^{\prime \prime}, \Delta \delta=50^{\prime \prime}, 23 \leq R<24$, solid-line contours and at $\Delta \alpha=60^{\prime \prime}$, $\Delta \delta=-50^{\prime \prime}, 24 \leq R<25$, dashed-line contours. For LBQS 1009-0252, a galaxy overdensity is found at $\Delta \alpha=60^{\prime \prime}, \Delta \delta=40^{\prime \prime}$ to quasar image A $(23 \leq R<25)$. For HE 2149-2745, the closest galaxy overdensity to the quasar images is centered at $\Delta \alpha=8^{\prime \prime}, \Delta \delta=-7^{\prime \prime}(23 \leq R<25)$.

Therefore, this cluster is most probably associated with the quasar $(z=2.33)$ rather than with the lens. The photometric redshift of the second galaxy overdensity has not been measured, being mostly outside the overlapping zone of the VLT and HST fields.

In the field around the six-image lensed quasar B 1359+154 we have measured the photometric redshifts of galaxies appearing in the overlapping VLT and HST fields (see Fig. 5). There are very few galaxies for which the SED is fitted with a $\chi^{2} \sim 1$ (16 galaxies among the 34 detected in the four bands). Half of them have a redshift between 1.1 and 1.5 and might correspond to the galaxy overdensity detected towards B 1359+154 because of their location in the field. Therefore, we will consider in the following, that the redshift of the galaxy cluster towards B $1359+154$ is $z=1.3$.

In the field around $\mathbf{H ~ 1 4 1 3 + 1 1 7}$ we have computed the photometric redshifts for galaxies located in the area covered by both the VLT and HST datasets. Two peaks are observed in redshift: a first one at $z=0.8 \pm 0.3$, and a second one, at $z=1.75 \pm 0.2$. As the two overdensities are mostly superimposed on the common HST and VLT frame, it is hard to identify their corresponding redshift. Yet, in the original paper reporting on the discovery of the South-East overdensity discovery (Kneib et al. 1998b), the photometric redshift derived 

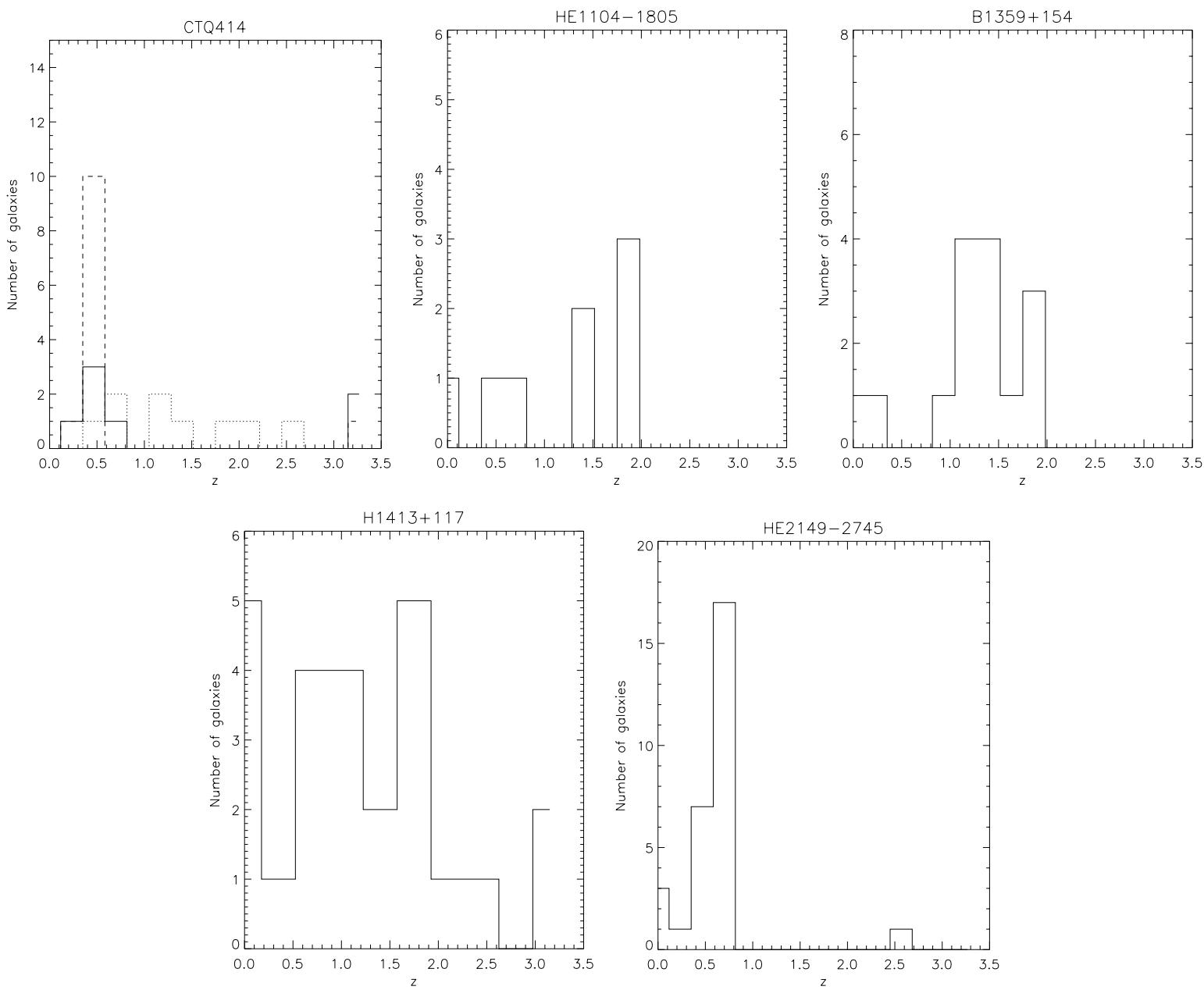

Fig. 5. Number of galaxies versus photometric redshifts for CTQ 414 (top left hand panel), for HE 1104-1805 (top central panel), for B 1359+154 (top right hand panel), for H 1413+117 (bottom left hand panel) and for HE 2149-2745 (bottom right hand panel). For CTQ 414, the solid-line corresponds to the photometric redshift distribution determined from the: $V, R, I, J$ and $K$ s galaxy magnitudes; the dotted-line corresponds to the redshift determination using the: $R, I, J$ and $K$ s galaxy magnitudes; the dash-line corresponds to the redshift determination using the: $V, R, J$ and $K$ s galaxy magnitudes.

(from three filters only) was $z=0.9 \pm 0.1$. We will assume in the following that the redshift of the South-East overdensity is $z=0.8 \pm 0.3$. The second galaxy overdensity could correspond either to the absorption system at $z=1.661$ or to that at $z=1.87$ (Turnshek et al. 1998; Magain et al. 1998.)

Finally, towards HE 2149-2745 the distribution of photometric redshifts (using the HST and VLT images) shows a peak in redshift at $z=0.7 \pm 0.1$ with a high confidence level. This suggests the presence of a galaxy cluster or galaxy group towards the doubly imaged quasar. On the other hand, previous studies by Wisotzki et al. (1998) and Lopez et al. (1998) argued that the lensing galaxy maximal redshift is $z=0.5$, according to its $V-R$ color. Due to the uncertainty on the photometric redshift determination and on the redshift limit given by Wisotzki et al., it is still uncertain whether the lensing galaxy is part of the galaxy cluster/group discovered in this study.

\subsection{Color versus magnitude diagrams}

We have analysed the $R-K$ s color versus $K$ s magnitude diagrams of the galaxies detected in the overlapping FORS1 and
ISAAC fields. Unfortunately, no elliptical red sequence appears in any of the field. The presence of such a sequence would argue in favor of the presence of a massive galaxy cluster towards the quasar. There are possibly two reasons for a nondetection: 1) there are too few galaxies in the common $R$ - and $K$ s-band catalogs (between 50 and 200). Hence, the elliptical red sequence of galaxy clusters does not show up in a conspicuous manner. 2) the galaxy clusters or groups are not massive enough to have an elliptical red sequence.

\section{Weak-shear analysis and mass reconstruction}

For each field, we have searched for the weak-shear signature that the galaxy groups or clusters should imprint on background galaxies. According to the gravitational lensing theory, the presence of a galaxy cluster or a galaxy group in the field generates distortions and amplifications of the background galaxies. In practice, we measure the "statistical" distortions: the mean ellipticity and mean position angle of the faintest galaxies in cells of a given size. 
Table 5. Results of the weak-shear analysis and of the mass reconstruction. Column 1: quasar name. Column 2: number of galaxies per $\operatorname{arcmin}^{2}$ corresponding to the galaxy overdensity. Column 3: position of the galaxy overdensity center relative to the quasar images. Column 4: photometric redshift of the galaxy overdensity and corresponding error bar (“*”: value inferred from previous paper, see text for references). Column 5: magnitude range for the background galaxies used for the weak-shear analysis. Column 6: number of galaxies used for the weakshear analysis and the mass reconstruction. Column 7: ICF width of the mass reconstruction in arcsecond. Column 8: upper limit of the mass integrated from the mass reconstruction in a radius of $500 \mathrm{kpc}$ and centered at the galaxy group position. Column 9: galaxy cluster velocity dispersion corresponding to the mass given in Col. 8. Column 10: shear orientation in degree.

\begin{tabular}{|c|c|c|c|c|c|c|c|c|c|}
\hline Quasar & $\begin{array}{l}\text { No. gal. } \\
/\left({ }^{\prime}\right)^{2}\end{array}$ & $\begin{array}{l}\text { Relative } \\
\text { position }\end{array}$ & $\begin{array}{l}z_{\text {phot }} \\
\pm \Delta z\end{array}$ & $\begin{array}{l}\text { Sources } \\
\text { mag. range }\end{array}$ & $\begin{array}{l}\text { No. gal } \\
\text { for WS }\end{array}$ & $\begin{array}{l}\text { ICF } \\
\text { (") }\end{array}$ & $\begin{array}{l}\text { Mass } \\
\left(10^{14} M_{\odot} \mathrm{h}^{-1}\right)\end{array}$ & $\begin{array}{l}\sigma_{v} \\
\left(\mathrm{~km} \mathrm{~s}^{-1} \mathrm{~h}^{-1}\right)\end{array}$ & $\mathrm{PA}_{\gamma}$ \\
\hline CTQ 414 & 28 & $12^{\prime \prime} \mathrm{SE}$ & $0.5 \pm 0.1$ & $25<R \leq 28$ & 578 & 180 & $<1.6$ & $<720$ & $24^{\circ}$ \\
\hline HE 0230-2130 & 21.1 & $38^{\prime \prime} \mathrm{W}$ & $1.0^{\star}$ & $25<R \leq 28$ & 633 & 120 & 4.0 & 1130 & $45^{\circ}$ \\
\hline LBQS 1009-0252 & _- & _ & $1^{\star}$ & $25<R \leq 28$ & 351 & 100 & $<2.4$ & $<880$ & $-33^{\circ}$ \\
\hline B $1030+074$ & 10 & $120^{\prime \prime} \mathrm{SE}$ & $0.6^{\star}$ & $23<R \leq 28$ & 416 & 120 & $<2.3$ & $<860$ & $33^{\circ}$ \\
\hline \multirow[t]{2}{*}{ HE 1104-1805 } & 9 & $40^{\prime \prime} \mathrm{N}$ & $2.0 \pm 0.1$ & $24<R \leq 28$ & - & - & - & - & $90^{\circ}$ \\
\hline & 18 & $78^{\prime \prime} \mathrm{SW}$ & - & - & - & - & _- & - & - \\
\hline B $1359+154$ & 4 & $7^{\prime \prime} \mathrm{NE}$ & $1.3 \pm 0.2$ & $23<R \leq 28$ & 749 & 300 & $<10.7$ & $<1800$ & $-12^{\circ}$ \\
\hline \multirow[t]{2}{*}{ H $1413+117$} & 43 & $14^{\prime \prime} \mathrm{SE}$ & $0.8 \pm 0.3$ & $25<R \leq 28$ & 453 & 100 & $<1.8$ & 760 & $45^{\circ}$ \\
\hline & 32 & $36^{\prime \prime} \mathrm{SW}$ & $1.7 \pm 0.2$ & _- & - & - & - & _- & - \\
\hline HE 2149-2745 & 22 & $7^{\prime \prime} \mathrm{SE}$ & $0.7 \pm 0.1$ & $25<R \leq 28$ & 81 & 60 & 3.2 & 1010 & $45^{\circ}$ \\
\hline
\end{tabular}

\subsection{PSF correction}

We have performed the weak-shear analysis on the FORS1/R-band images of the systems. First of all, we have to correct the galaxy ellipticities for the instrumental distortions across the field. Each night, the PSF variations across the field are measured from a sample of stars, using the Im2shape software (Bridle et al. 2002; Kneib et al. 2003). The ellipticity of the PSF varies typically from $\epsilon=(a-b) /(a+b)=0.002$ to 0.05 across the FORS 1 field. Then, the background galaxies are deconvolved in an analytical way, modeling them by 2D-functions and using the closest PSF.

\subsection{Distorsion measurement}

Once the galaxy images have been deconvolved and using only galaxies with ellipticities known with an accuracy better than $20 \%$, we can compute mean galaxy ellipticities and mean galaxy orientations across the field. The field is divided into a grid of cells. In order to optimize the coherence of the shear-map pattern, we attribute to any galaxy within the cell an ellipticity and an orientation derived through averaging the ellipticities and the orientations of all galaxies within a radius $r$ slightly larger than half the cell size. Then, within each cell, the attributed ellipticities and attributed orientations are averaged for all the galaxies. These final "mean ellipticity" and "mean orientation" are allocated to the cell center. In a field

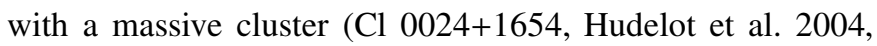
private communication) we have tested that, for the total number of galaxies used to perform the weak-shear analysis (between 1000 and 2500 galaxies per field), a combination of a cell $\sim 40^{\prime \prime} \times 40^{\prime \prime}$, and a radius $r$ of $0.01^{\circ}$ to $0.02^{\circ}$, allows to extract the statistical information in a satisfactory way.

None of the shear map of these systems reveals a signal strong enough to be spotted at first glance. However the shear map can be used to derive the lensing potential mass distribution as well as an upper limit of its mass. This is detailed in the following subsection.

\subsection{Mass reconstruction}

The galaxy mean ellipticities and mean orientations are given as input to the LensEnt2 software (Bridle et al. 1998; Marshall et al. 2002), a code which allows to built the mass distribution of a lens using the shear-map. The redshift of the lens plane assumed for this reconstruction is that provided in Table 5 (Col. 4). The source plane redshift is derived from the photometric redshift of the FDF galaxies (E. Labbe, private communication, see Heidt et al. 2003 for the photometry): in a given magnitude range (displayed in Table 5), we have averaged the FDF galaxy photometric redshifts and assigned this mean redshift to the galaxy sample. For all magnitude ranges considered here, the mean galaxy redshift is $z \sim 1.4$. The reconstruction is performed in a frame 4 times larger than the frame where the weak-shear was analysed since the shear pattern eventually observed in the FORS1 field could be due to the presence of a mass potential located outside the field of view. LensEnt 2 uses a maximum entropy method to reconstruct the mass potential. The program simulates a synthetic population of unlensed background galaxies at a same redshift and in a field of the same size as the observed one (Bartelmann et al. 1996). Then LensEnt 2 adds in the field a mass clump, with a compactness characterized by its Intrinsic Correlation Function (ICF, Marshall et al. 2002), at the lens redshift, and analyses the similarity between this simulated shear pattern and the observed shear pattern in each field.

The ICF of the mass clump is assumed to be Gaussian. For each system, we have performed a set of mass reconstructions using different ICF widths. The "evidence" (see Marshal et al. 2002) determined for each mass reconstruction provides a good discriminator of the ICF width, weighting the reality of the 

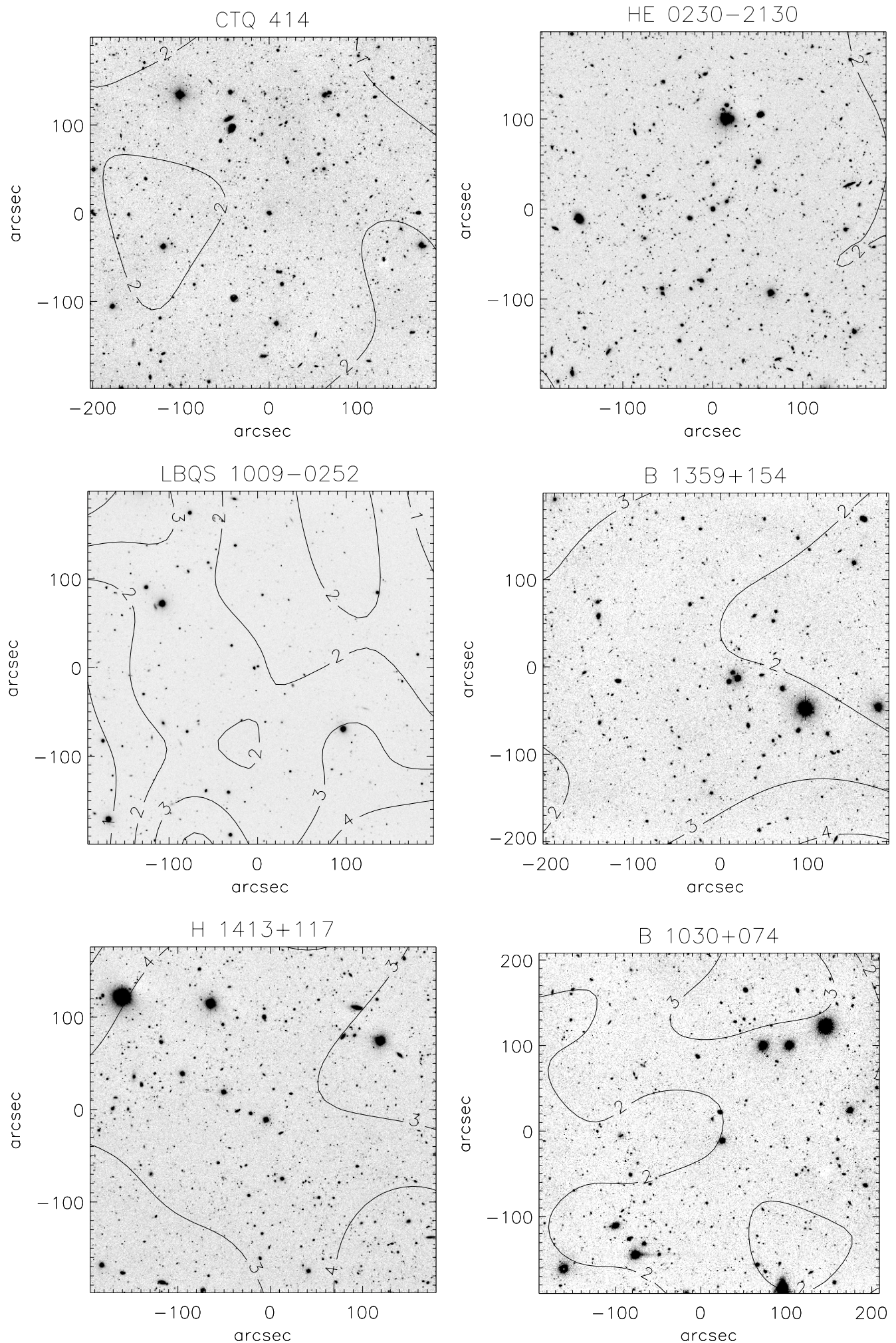

Fig. 6. Mass reconstruction superimposed on the FORS1 images. The contours outline regions of same signal-to-noise (dash-lines: $S / N<1$, solid-lines: $S / N \geq 1$ ). From the top left hand corner to the bottom right hand corner: a) CTQ 414, b) HE 0230-2130, c) LBQS 1009-0252, d) B $1359+154$ and e) $\mathrm{H} 1413+117$.

obtained mass reconstruction. The width of the ICF finally selected is given in Table 5. Typically, the mass bump which reveals a galaxy cluster in a reconstruction map corresponds to an ICF width between $100^{\prime \prime}$ and $300^{\prime \prime}$.
Since the noise properties of maximum-entropy inversion methods are not easy to interpret, we have performed, for each system, 80 reconstructions starting with modified catalogs of galaxies (positions are retained but ellipticity directions are 
assigned at random). For each of the 80 mocked catalogs, mass reconstruction is performed, and a set of mass bumps is obtained. Integrating over the 80 catalogs, the mass bump distribution shows a number of peaks. Their median gives the noise level of this procedure. Results of the mass reconstructions towards the systems are displayed in Fig. 6. The contours outline regions of same signal to noise ratio. The signal to noise of the mass reconstruction is low at the location of the galaxy overdensity $(S / N<3$ in all frames), and there are regions in the field with higher mass signal than at the position of the galaxy overdensity. The low signal at the overdensity location could have different origins: 1 ) there is actually no mass clump at this position 2) the mass inferred in the fields are too low to be detected by the reconstruction, and therefore the masses measured here are upper limit 3) the largest structures in the field (real or artifacts due to the low number of background galaxies in some cases) are adding noise, and the signal level at the overdensity position is biased. Therefore, we investigate the limit of the LensEnt 2 software performing the following test: for each system, we have lensed the background galaxies with a Pseudo Isothermal Elliptical Sphere (Kassiola \& Kovner 1993) of small ellipticity $\left(\epsilon=\left(a^{2}-b^{2}\right) /\left(a^{2}+b^{2}\right)=0.05\right)$ and centered on the quasar image A. We have looked for the smallest mass potential that the software can reconstruct. This value varies in each field as the number of background galaxies is different. The lower mass values (in a radius of $500 \mathrm{kpc}$ ) are displayed in Table 6 (Col. 3), as well as the mass integrated in a same radius, but centered on the overdensity in the original mass map.

\subsection{Results}

The sizes of the mass clumps computed by the program, as well as the masses inferred, correspond to small galaxy clusters, or even galaxy groups.

In Table 5, the mass displayed are integrated over a radius of $500 \mathrm{kpc}$ (typical size for a galaxy cluster) and centered on the galaxy overdensity. The velocity dispersion is derived from the mass value, assuming that the mass distribution follows an Isothermal Sphere potential with a core radius of $30 \mathrm{kpc}$.

We have chosen to rebuild the mass towards LBQS 1009-0252 even though no evidence for the presence of any galaxy cluster towards the quasar has been found. We choose arbitrarily $z=1$ for the lensing potential redshift and a galaxy magnitude range of $R=$ [23, 28[ for the measurement of distortions. The lack of mass concentration confirms that we do not detect any extra gravitational potential in the field around LBQS 1009-0252.

From the results of the mass limit test displayed in Table 6 , we see that the only systems for which the mass inferred $M$ have a physical relevancy are HE 2149-2745 and HE 0230-2130, as they appear to be larger than the detection

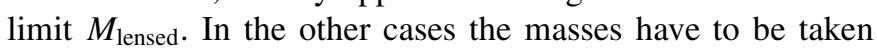
as upper mass limits. In the case of LBQS 1009-0252 the mass measured is well below the detection limit, and this suggest that the overdensity detected here is not related to a mass potential.
Table 6. Galaxy group masses. Column 1: quasar name. Column 2: mass integrated in the original mass map, in a radius of $500 \mathrm{kpc}$ centered on the galaxy overdensity. Column 3: lower mass value reconstructible by LensEnt 2 .

\begin{tabular}{cll}
\hline \hline & $M(<500 \mathrm{kpc})$ & $M_{\text {lensed }}(<500 \mathrm{kpc})$ \\
& $10^{14} M_{\odot} h^{-1}$ & $10^{14} M_{\odot} h^{-1}$ \\
\hline CTQ 414 & 1.6 & 1.7 \\
HE 0230-2130 & 4.0 & 3.6 \\
LBQS 1009-0252 & 2.4 & 5.3 \\
B 1030+074 & 2.3 & 2.4 \\
B 1359+154 & 10.7 & 12.7 \\
H 1413+117 & 1.8 & 2.0 \\
HE 2149-2745 & 3.2 & 2.6 \\
\hline
\end{tabular}



Fig. 7. Shear strength induced by the galaxy groups towards the different systems as a function of $\mathrm{H}_{0}$.

Finally, the LensTool software provides a map of the shear induced by a mass distribution according to the distance to the potential center. Therefore, we can easily extract the shear strengths that the galaxy groups induce at the quasar positions. These values are displayed in Fig. 7 versus the value of the Hubble constant.

The shears computed here are derived from the masses of the galaxy groups. They are, as a consequence, shear upper limit values in the same case of CTQ 414, B 1030+074, B 1359+154 and H 1413+117. We notice that they are in general lower than those predicted in former studies (displayed in 
Table 1). Moreover the orientations are also different from the predicted ones. These discrepancies between our results and previous ones might come from the fact that the predicted shear values and orientations had been formerly deduced by modeling the lensing galaxy as a sphere, that is to say without taking into account its orientation and ellipticity, while these last parameters dominate the shear strength and orientation in most cases.

\section{Conclusions}

We have studied the surroundings of the quasar images in eight gravitationally lensed systems in order to search for the presence of galaxy clusters or galaxy groups which could contribute to the "external shear" requested from previous studies. We have used three different and independent methods: the search of galaxy number overdensities, the search of a peak in the redshift distribution of galaxies - using photometric redshifts -, and finally a weak-shear analysis combined with mass reconstruction of the background galaxies.

There is convincing evidence for the presence of a galaxy group in the line of sight towards the following 5 lensed quasars: CTQ 414, HE 0230-2130, B 1359+154, H 1413+117 and HE 2149-2745. Towards LBQS 1009-0252 and B 1030+074 we have not been able to compute the photometric redshift of the overdensity, and, therefore, we cannot conclude about their 3D reality. The galaxy overdensity discovered in the field around HE 1104-1805 seems to be associated with the quasar rather than with the lensing potential.

The mass reconstruction leads to small mass clump sizes, related to galaxy groups. Moreover, the galaxy group masses and the shear they induce at the quasar position have to be regarded as upper limits, as we are using the reconstruction mass method at the edge of its applicability.

The next step of this work is to incorporate the information derived from the wide-field analyses in the modeling of the lensing potential towards these eight lensed quasars. This will ultimately lead to an improved value for the Hubble constant, as derived from available time-delay measurements between the quasar image light-curves.

Acknowledgements. The HST data used in this paper were obtained by the "CfA Arizona Space Telescope LEns Survey" (CASTLES) collaboration (PI: E. Falco). C.F. acknowledges support from an ESO studentship in Santiago and a grant from the "Société de Secours des Amis des Sciences". F.C. is supported by the European Comission through Marie Curie grant MCFI-2001-0242. The ECOS/CONICYT grant CU00U05 is also gratefully acknowledged. J-P.K. acknowledges support from Caltech and CNRS. F.C. is partially funded by the "Pôle d'Attraction Interuniversitraire" P4/05 (STSC, Belgiun).

\section{References}

Amico, P., Cuby, J. G., Devillard, N., et al. 2002, ISAAC Data Reduction Guide 1.5

Angonin-Willaime, M.-C., Soucail, G., \& Vanderriest, C. 1994, A\&A, 291,411
Bardeau, S., Kneib, J.-P., Czoske, O., et al. 2004, in preparation

Bartelmann, M., Narayan, R., Seitz, S., \& Schneider, P. 1996, ApJ, 464, L115

Bertin, E., \& Arnouts, S. 1996, A\&AS, 117, 393

Bolzonella, M., Miralles, J.-M., \& Pelló, R. 2000, A\&A, 363, 476

Bridle, S., Hopson, M., Saunders, R., \& Lasenby, A. 1998, MNRAS, 299, 895

Bridle, S., Kneib, J.-P., Bardeau, S., \& Gull, S. F. 2002, in The shapes of Galaxies and their Dark Halos, Yale Cosmology workshop, 28-30 May 2001 (World Scientific)

Browne, I., Patnaik, A., Wikinson, P., \& Wrobel, J. 1998, MNRAS, 293, 257

Burud, I., Courbin, F., Magain, et al. 2002, A\&A, 383, 71

Calzetti, D., Armus, L., Bohlin, R. C., et al. 2000, ApJ, 533, 682

Chartas, G., Gupta, V., Garmire, G., et al. 2002, ApJ, 565, 96

Claeskens, J.-F., Khmil, S., Donk Wook Lee Sluse, D., \& Surdej, J. 2001, A\&A, 367, 748

Courbin, F., Lidman, C., \& Magain, P. 1998, A\&A, 330, 57

Courbin, F., Lidman, C., Meylan, G., Kneib, J.-P., \& Magain, P. 2000, A\&A, 360, 853

Cuby, J. G., Lidman, C., Moutou, C., et al. 2002, ISAAC handbook, ESO webpage

Fassnacht, C., \& Cohen, J. 1998, AJ, 115, 377

Faure, C., Courbin, F., Kneib, J. P., et al. 2002, A\&A, 386, 69

Faure, C., Alloin, D., Gras, S., et al. 2003, A\&A, 405, 415

Faure, C., Alloin, D., Courbin, F., \& Kneib, J.-P. 2004, A\&A, in preparation

Gil-Merino, R., Wisotzki, L., \& Wambsganss, J. 2002, A\&A, 381, 428

Grant, C., Bautz, M., Chartas, G., \& Garmire, G. 2003, ApJ, in press [arXiv: astro-ph/0305137]

Hazard, C., Morton, D. C., Terlevich, R., \& McMahon, R. 1984, ApJ, $282,33 \mathrm{H}$

Heidt, J., Appenzeller, I., Gabasch, A., et al. 2003, A\&A, 398, 49

Hewett, P., Irwin, M., Foltz, C., et al. 1994, AJ, 108, 1534

Hudelot, P., Kneib, J.-P., et al. 2004, in preparation

Jackson, N., Xanthopoulos, E., \& Browne, I. 2000, MNRAS, 311, 389

Kassiola, A., \& Kovner, I. 1993, ApJ, 417, 450

Keeton, C., \& Kochanek, C. 1998, ApJ, 495, 157

Keeton, C. R., Kochanek, C. S., \& Seljak, U. 1997, ApJ, 482, 604

Keeton, C., Kochanek, C., \& Falco, E. 1998, ApJ, 509, 561

Kochanek, C. 1996, ApJ, 466, 638

Kneib, J.-P., Alloin, D., Mellier, Y., et al. 1998a, A\&A, 329, 827

Kneib, J.-P., Alloin, D., \& Pello, R. 1998b, A\&A, 339, 65

Kneib, J.-P., Cohen, J., \& Hjorth, J. 2000, ApJ, 544, L35

Kneib, J.-P., Hudelot, P., Ellis, R., et al. 2003, ApJ, 598, 804

Kundic, T., Cohen, J., Blandford, R., \& Lubin, L. 1997, AJ, 114, 507

Lehàr, J., Falco, E., Kochanek, C., et al. 2000, ApJ, 536, 584

Lidman, C., Courbin, F., Kneib, J.-P., et al. 2000, A\&A, 364, L62

Lopez, S., Wucknitz, O., \& Wisotzki, L. 1998, A\&A, 339, L13

Magain, P., Surdej, J., Swing, J.-P., et al. 1988, Nature, 334, 325

Marshall, P. J., Hobson, M. P., Gull, S. F., \& Bridle, S. L. 2002, MNRAS, 335, 1193

Maza, J., Wischnjewsky, M., Antezana, R., \& Gonzalez, L. E. 1995, Rev. Mex. Astron. Astrofis., 31, 119M

Morgan, N., Dressler, A., Maza, J., Schechter, P., \& Winn, J. 1999, AJ, 118,1444

Morgan, N., Chartas, G., Malm, M., et al. 2001, ApJ, 555, 1M

Muñoz, J., Falco, E., Kochanek, C., et al. 1998, Ap\&SS, 263, 51M

Ofek, E., \& Maoz, D. 2003, ApJ, 594, 101

Patnaik, A. 1993, Proc. 31st Liège International Astrophys. Coll. Gravitational lenses in the Universe, 311

Patnaik, A., Browne, I., Wilkinson, P., \& Wrobel, J. 1992, MNRAS, 254,655 
Pelt, J., Refsdal, S., \& Stabel, R. 2002, A\&A, 389, L57

Refsdal, S. 1964, MNRAS, 128, 307

Remy, M., Claeskens, J.-F., Surdej, J., et al. 1998, NewA, 3, 379

Rusin, D., Kochanek, C., Norbury, M., et al. 2001, ApJ, 557, 594

Saha, P., \& Williams, L. 2003, AJ, 125, 2769S

Schneider, P. 1997, rpa work, 33

Schechter, P., Udalski, A., Szymanski, M., et al. 2003, ApJ, 584, 657

Surdej, J., Remy, M., Smette, A., et al. 1993, Proc. 31st Liège International Astrophys. Coll. Gravitational Lenses in the Universe, 153

Szeifert, T. 2002, FORS handbook, ESO webpage

Wilkinson, P., Browne, I., Patnaik, A., Wrobel, J., \& Sorathia, B. 1998, MNRAS, 300, 790
Turnshek, D. A., Foltz, C. B., Grillmair, C. J., \& Weyman, R. J. 1998, ApJ, 325, 651

Wisotzki, L., Kohler, T., Kayser, R., \& Reimers, D. 1993, A\&A, 278, L15

Wisotzki, L., Kohler, T., Groote, D., \& Reimers, D. 1996, A\&A, 115, 27

Wisotzki, L., Wucknitz, O., Lopez, S., \& Norup Sorensen, A. 1998, A\&A, 339, 73

Wisotzki, L., Chriestlieb, N., Liu, M. C., et al. 1999, A\&A, 348, 41

Xanthopoulos, E., Browne, I., King, L., et al. 1998, MNRAS, 300, 649

Yamada, T., Tanaka, I., Aragon-Salamanca, A., et al. 1997, ApJ, 487, 125 